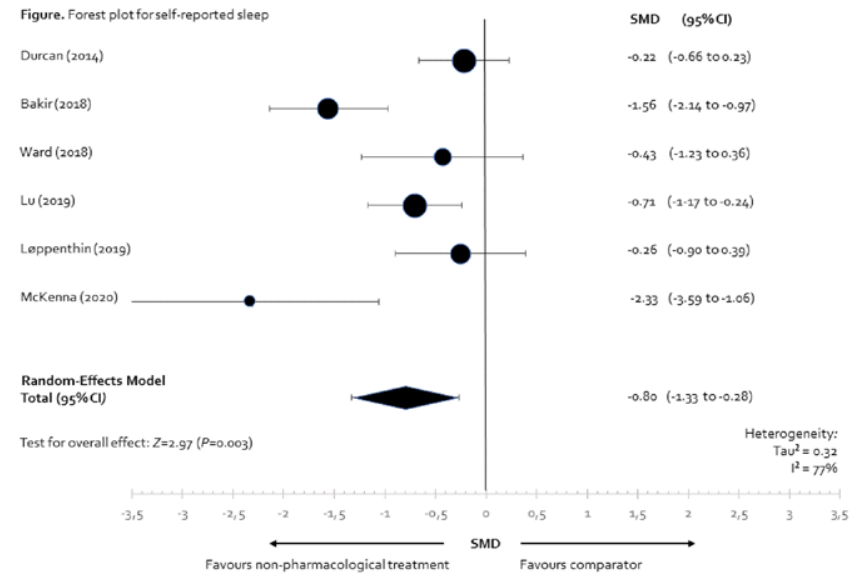

Conclusion: Although the effect of non-pharmacological interventions targeting sleep disturbances or the sleep disorder insomnia was statistically highly significant, the implication for clinical practice is questionable because of the overall quality evidence. None of the core outcomes used in contemporary IA trials have indicated clinical benefit in favour of non-pharmacological interventions targeting sleep disturbances or disorders.

In conclusion, more rigorous research on non-pharmacological management of sleep disturbances and disorders is urgently needed, also aimed at specific sleep disorders, in order to fully reveal the clinical utility of these novel treatment options. At this point, non-pharmacological treatment of sleep disturbances or disorders is promising and potentially highly effective, and may have the potential to persistently decrease the symptom burden and increase the quality of life of patients with IA.

REFERENCES:

[1] Li et al., Psychol Health Med. 2019 Sep;24(8):911-924

[2] Haugeberg et al., Arthritis Res Ther. 2020 Aug 26;22(1):198

[3] Wali et al., J Clin Sleep Med. 2020 Feb 15;16(2):259-265

[4] Løppenthin et al., Clin Rheumatol. 2015 Dec;34(12):2029-39

Disclosure of Interests: Kristine Marie Latocha: None declared, Katrine Løppenthin: None declared, Safa Al-Bazy: None declared, Tannie Albrechtsen: None declared, Helle Jensen: None declared, Mikkel Østergaard Speakers bureau: Abbvie, Celgene, Eli-Lilly, Janssen, Novartis, Pfizer, Roche, Sanofi and UCB, Consultant of: Abbvie, BMS, Boehringer-Ingelheim, Celgene, Eli-Lilly, Hospira, Janssen, Merck, Novartis, Novo, Orion, Pfizer, Regeneron, Roche, Sandoz, Sanofi and UCB, Grant/research support from: Abbvie, BMS, Merck, Celgene, Novartis, Poul Jennum: None declared, Bente Appel Esbensen: None declared, Robin Christensen: None declared

DOI: 10.1136/annrheumdis-2021-eular.836

\section{OP0160-HPR PREFERENCES FOR TREATMENTS TO PREVENT RHEUMATOID ARTHRITIS: DISCRETE CHOICE SURVEY OF GENERAL POPULATIONS IN UNITED KINGDOM, GERMANY, AND ROMANIA}

G. Simons ${ }^{1}$, J. Veldwijk2 ${ }^{2}$, R. DI Santostefano ${ }^{3}$, M. Englbrecht ${ }^{4}$, C. Radawski ${ }^{5}$, L. Valor ${ }^{6}, \mathrm{~K}$. Raza $^{7}$, M. Falahee ${ }^{7}{ }^{1}$ University of Birmingham, Rheumatology Research Group, Institute of Inflammation and Ageing, Birmingham, United Kingdom; ${ }^{2}$ Erasmus University Rotterdam, Erasmus School of Health Policy and Management, Rotterdam, Netherlands; ${ }^{3} J a n s s e n ~ R \& D$, Janssen R\&D, NJ, United States of America; ${ }^{4}$ Freelance data scientist, Freelance data scientist, Eckental, Germany; ${ }^{5}$ Eli Lilly \& Company, Eli Lilly \& Company, Indianapolis, United States of America; ${ }^{6}$ Friedrich-Alexander-University Erlangen, Department of Internal Medicine, Erlangen, Germany; ${ }^{7}$ University of Birmingham, Rheumatology Research Group, Institute of Inflammation and Ageing, Birmingham, United Kingdom

Background: There is increasing research focus on intervention for rheumatoid arthritis (RA) at the earliest stages of disease development, including treatment to prevent RA in at-risk groups. Novel cellular therapies are in development, and the effectiveness of existing immunomodulatory agents to prevent RA in those at risk is under investigation. Quantitative evidence of likely uptake of preventive treatments, and preferences for benefits and risks of such treatments is limited. Objectives: To quantify preferences for preventive therapies for RA.

Methods: A web-based survey $(n=2959)$ was administered to an age- and gender- stratified sample of adults in the general population from online survey panels in the UK, Germany, and Romania. After receiving information about RA, questions to check comprehension of background information, an introduction to the survey tasks and warm-up questions, participants were asked to imagine that they were experiencing arthralgia (without swelling) and had positive autoantibody tests indicating a $60 \%$ chance of developing RA in the next two years. Using a discrete choice experiment with a Bayesian D-efficient design, participants were offered a series of 15 choices between no treatment and two unlabeled hypothetical treatments to lower risk of RA development. Treatments were defined by six attributes with varying levels including benefits, risks, and frequency/route of administration (Table 1). One choice task with fixed levels described treatments representative of those under investigation for RA prevention (abatacept, hydroxychloroquine, atorvastatin and tolerogenic cell-based therapy). Attribute selection and presentation was informed by previous qualitative research, ranking surveys, systematic literature review, and expert opinion. Survey design was informed by patient research partners. The survey was pretested during qualitative interviews and revised. A pilot of the final survey with 100 respondents was conducted to obtain priors for the final experimental design. Random parameters logit (RPL) models were used to estimate relative importance of treatment attributes and likely treatment uptake rates in each country.

Table 1. Treatment attributes and levels

\begin{tabular}{ll}
\hline Attribute & \multicolumn{1}{c}{ Levels } \\
\hline $\begin{array}{ll}\text { Chance of developing RA reduced from } 60 \% \text { to } \\
\text { How the treatment is taken }\end{array}$ & $\begin{array}{l}10 \% ; 20 \% ; 30 \% ; 40 \% \\
\text { A shallow injection } \\
\text { under the skin } \\
\text { A drip into the vein } \\
\text { One or two tablets } \\
\text { Daily }\end{array}$ \\
How often the medication has to be taken & Weekly \\
& Monthly \\
& Every 6 months \\
Chance of mild side effects & $2 \% ; 5 \% ; 10 \%$ \\
Chance of a serious infection due to treatment & $0 \% ; 1 \% ; 5 \%$ \\
Chance of a serious side effect that is potentially irreversible & 1 in 100,000 people \\
& 20 in 100,000 people \\
& 100 in 100,000 people
\end{tabular}

Results: Across all three countries, effectiveness was the treatment attribute that had most impact on treatment choice (Figure 1). Method of administration was second most important for respondents from the UK and Romania but less important for German respondents. Risks of serious infection and serious side effects were more important determinants of treatment choice for respondents in Romania than they were in the UK and Germany. Percentage choice of fixed profiles reflecting abatacept, atorvastatin, hydroxychloroquine, tolerogenic cellbased therapy and no treatment differed across countries $\left(\chi^{2}=78.90 ; p<0.001\right)$ : $28.3 \%, 20.6 \%, 22.2 \% 18.5 \%$ and $10.4 \%$ respectively in the UK; $31.3 \%, 18.8 \%$, $11.2 \%, 23.4 \%$ and $15.3 \%$ in Germany; and $27.6 \%, 20.5 \%, 15.8 \%, 21.7 \%$ and $14.4 \%$ in Romania.

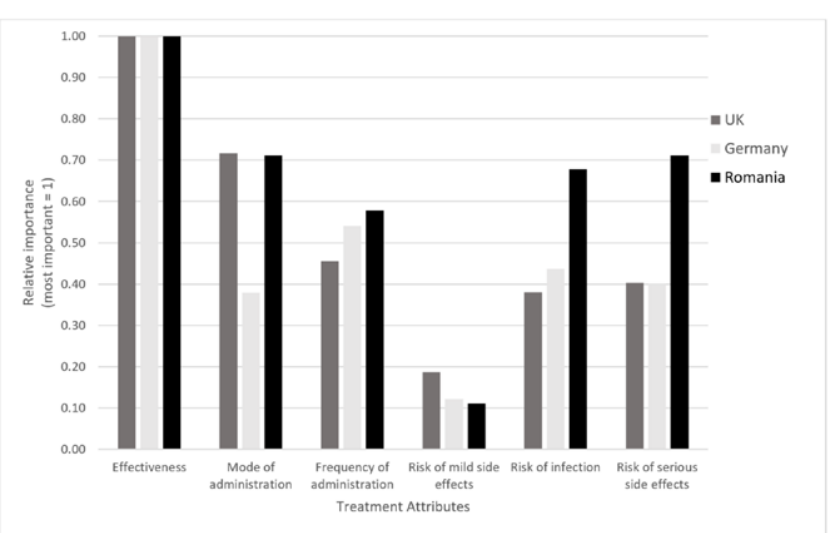

Figure 1: Relative importance of treatment attributes stratified by country; UK ( $N=982$ ), Germany $(N=984)$ and Romania $(N=993)$. Separate RPL models were used for each country.

Conclusion: This study suggests that effective preventive treatments for RA are acceptable to members of the general population told to assume up a $60 \%$ chance of developing RA. The relative importance of treatment attributes and likely uptake of fixed treatment profiles differed across countries. These findings are informative for the design of prevention trials, and the development of informational resources and efficient preventive strategies for those at risk of developing RA.

Acknowledgements: On behalf of the PREFER project. PREFER received funding from the IMI 2 Joint Undertaking (grant No. 115966), which receives support from the EU's Horizon 2020 research and innovation program and European Federation of Pharmaceutical Industries and Associations (EFPIA). K. Raza is supported by the NIHR Birmingham Biomedical Research Centre. 
Disclosure of Interests: Gwenda Simons: None declared, Jorien Veldwijk: None declared, Rachael Di Santostefano Shareholder of: Johnson \& Johnson, Employee of: Janssen R\&D (of Johnson \& Johnson), Matthias Englbrecht Speakers bureau: AbbVie, Chugai, Eli Lilly, Novartis, Roche, Sanofi, Mundipharma, Paid instructor for: AbbVie, Chugai, Roche, Consultant of: AbbVie, Novartis, Roche, Sanofi, Grant/research support from: Roche, Chugai, Christine Radawski Shareholder of: Eli Lilly \& Company, Employee of: Eli Lilly \& Company, Larissa Valor: None declared, Karim Raza Consultant of: Personal fees from Abbvie, Pfizer, Sanofi, Lilly, Bristol Myers Squibb, UCB, Janssen, and Roche Chugai, Grant/research support from: Abbvie and Pfizer, M. Falahee: None declared DOI: 10.1136/annrheumdis-2021-eular.2168

\section{Advances in Pediatric Rheumatology}

\begin{tabular}{|l|l|}
\hline OP0161 & PREDICTIVE VALUE OF MUSCULOSKELETAL \\
ULTRASOUND IN PATIENTS WITH JUVENILE \\
IDIOPATHIC ARTHRITIS IN CLINICAL REMISSION
\end{tabular}

M. Mazzoni ${ }^{1}$, S. Merlo ${ }^{1,2}$, C. Morreale ${ }^{1,2}$, A. Pistorio $^{3}$, S. Viola ${ }^{2}$, S. Ancona ${ }^{1,2}$, F. Magnaguagno ${ }^{4}$, A. Consolaro ${ }^{1,2}$, A. Ravelli ${ }^{1,2}$, C. Malattia ${ }^{1,2}{ }^{1}$ Università degli Studi di Genova, Dipartimento di Neuroscienze, Riabilitazione, Oftalmologia, Genetica e Scienze Materno-Infantili, Genoa, Italy; ${ }^{2}$ IRCCS Istituto Giannina Gaslini, Clinica Pediatrica e Reumatologia, Genoa, Italy; ${ }^{3}$ IRCCS Istituto Giannina Gaslini, Epidemiologia e Biostatistica, Genoa, Italy; ${ }^{4}$ RCCS Istituto Giannina Gaslini, UOC Radiologia, Genoa, Italy

Background: The accurate assessment of remission status in JIA patients is of utmost relevance to taper medications and prevent side effects from their longterm administration. In RA patients in clinical remission (CR), musculoskeletal ultrasound (MSUS) allows to detect persistent joint inflammation (subclinical synovitis), which predicts disease flare and structural damage progression. Although subclinical synovitis has been reported in a substantial proportion of JIA patients with inactive disease, its prognostic value is still being defined. Objectives: 1) to investigate the prevalence of MSUS-detected subclinical synovitis in JIA patients in CR; 2) to establish which and how many joints should be scanned to reliably assess remission; 3 ) to evaluate the persistence of subclinical synovitis over the time; 4 ) to investigate whether subclinical synovitis entails a risk of disease flare and whether it should affect the therapeutic strategy.

Methods: 135 consecutive JIA patients who met the Wallace criteria for CR were included in this 3-years prospective study. All patients underwent MSUS assessment of 56 joints at study entry and at 6 months follow-up visit. Joints were scanned for synovial hyperplasia, joint effusion and Power Doppler (PD) signal by two independent ultrasonographers. Patients were followed clinically for 3 years. A flare of synovitis was defined as a recurrence of clinically active arthritis. The association between clinical and MSUS variables with flare, was evaluated by adjusted logistic regression models.

Results: 135 patients ( $78.5 \%$ F; median age 11.3 y; median disease duration 5.7 $\mathrm{y}$; median CR duration $1.4 \mathrm{y}$ ) were included. Fifty-seven/135 (42.2\%) patients had persistent oligoarthiritis; 41/135 (30.4\%) extended oligoarthiritis; 32/135 (23.7\%) polyarthiritis; $5 / 135(3.7 \%)$ systemic arthritis. Seventy-eight/135 (57.7\%) patients were in CR on medication. Subclinical synovitis was detected in 32/135 (23.7\%) patients and in $53 / 7560(0.7 \%)$ joints. Subclinical tenosynovitis was present in $20 / 135(14.8 \%)$ patients. Subclinical synovitis was found more frequently in the ankle and wrist joints. $58.6 \%$ of patients showed persistent subclinical synovitis at 6 month follow up MSUS examination. During the 3-year follow up 45/135 (33.3\%) patients experienced a disease flare (median survival time $2.2 \mathrm{y}$ ). PD positivity in tendons was the stronger independent risk factor of flare on multivariable regression analysis (HR: $4.8 ; \mathrm{P}=0.04)$. Other predictors of flare were the JIA subtype (oligo-extended form: HR: 2.3; $\mathrm{P}=0.031$ ) and the status of $C R$ on medication (HR: $3.7 ; \mathrm{P}=0.002$ ).

Conclusion: our results confirm that MSUS is more sensitive than clinical evaluation in the assessment of persistent synovial inflammation in JIA patients. Subclinical tenosynovitis was the best predictor of disease flare. To date, the role of tenosynovitis in the diagnosis and prognosis of JIA has been poorly investigated. Our results further support the role of MSUS, especially of the wrist and the ankle, in monitoring JIA patients in clinical remission and to predict disease flare. REFERENCES:

[1] De Lucia O, et al. Baseline ultrasound examination as possible predictor of relapse in patients affected by juvenile idiopathic arthritis (JIA). Ann Rheum Dis. 2018 Oct;77(10):1426-1431.

[2] Filippou G, et al. The predictive role of ultrasound-detected tenosynovitis and joint synovitis for flare in patients with rheumatoid arthritis in stable remission. Results of an Italian multicentre study of the Italian Society for Rheumatology Group for Ultrasound: the STARTER study. Ann Rheum Dis 2018;77:1283-9
Disclosure of Interests: None declared

DOI: 10.1136/annrheumdis-2021-eular.3823

\section{OP0162 IDIOPATHIC ARTHRITIS}

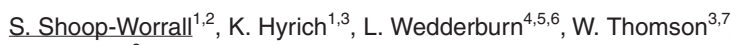

N. Geifman ${ }^{2}$ on behalf of BSPAR-ETN Study, BCRD Study, CAPS, CHARMS,

CLUSTER. ${ }^{1}$ The University of Manchester, Centre for Epidemiology Versus Arthritis, Manchester, United Kingdom; ${ }^{2}$ The University of Manchester, Centre for Health Informatics, Manchester, United Kingdom; ${ }^{3}$ Manchester University NHS FT, Manchester Academic Health Science Centre, NIHR Manchester BRC, Manchester, United Kingdom; ${ }^{4}$ UCL Great Ormond Street Institute of Child Health, Centre for Adolescent Rheumatology Versus Arthritis, London, United Kingdom; ${ }^{5}$ Great Ormond Street Hospital NHS Foundation Trust, Paediatric Rheumatology, London, United Kingdom; ${ }^{6}$ Great Ormond Street Biomedical Research Centre, NIHR, London, United Kingdom; ${ }^{7}$ The University of Manchester, Centre for Genetics and Genomics Versus Arthritis, Manchester, United Kingdom

Background: In children and young people (CYP) with JIA, we have previously identified clusters with different patterns of disease impact following methotrex ate (MTX) initiation. It is unclear whether clusters of treatment response following etanercept (ETN) therapy exist and whether, in a group of CYP who have responded inadequately to or had adverse events on methotrexate, similar treatment response patterns exist. Novel response patterns would aid stratified treatment approaches through better understanding and potential forecasting of more specific response patterns across multiple domains of disease.

Objectives: To identify and characterise trajectories of juvenile arthritis disease activity score (JADAS) components following ETN initiation for JIA.

Methods: ETN-naïve CYP with non-systemic JIA were selected if enrolled prior to January 2019 in at least one of four CLUSTER consortium studies: BSPARETN, BCRD, CAPS and CHARMS, at point of starting ETN as their first biological therapy. JADAS components (active joint count, physician's global assessment $(0-10 \mathrm{~cm})$, parental global evaluation $(0-10 \mathrm{~cm})$ and standardised ESR $(0-10)$ were collected at ETN initiation and during the following year.

Multivariate group-based trajectory models, that identify clusters of CYP with similar patterns of change over time, were used to explore ETN response clusters across the different JADAS components. Censored-normal (global scores, ESR) and zero-inflated Poisson (active joint count) models were used, adjusting for year of ETN initiation. Optimal models were selected based on a combination of model fit (BIC), parsimony, and clinical plausibility.

Results: Of the 1003 CYP included, the majority were female $(70 \%)$ and of white ethnicity $(90 \%)$, with rheumatoid factor-negative JIA the most common disease category $(39 \%)$.

The optimal model identified five trajectory clusters of disease activity following initiation of ETN (Figure 1). Clusters following ETN were similar and covered similar proportions of CYP to those previously identified following MTX: Fast (Group 1: $13 \%$ ) and Slow (Group 2: 10\%) response, active joint count improves but either physician (Group 3: 6\%) or parent global scores (Group 4: 34\%) remain persistently raised and a group with persistent raised scores across all JADAS com ponents (Group 5: 36\%). Compared to the persistent disease cluster, those with greater improvement had lower age and higher functional ability at ETN initiation and those with persistent raised parent global scores had lower ESR levels and were less likely to be RF-positive at ETN initiation.
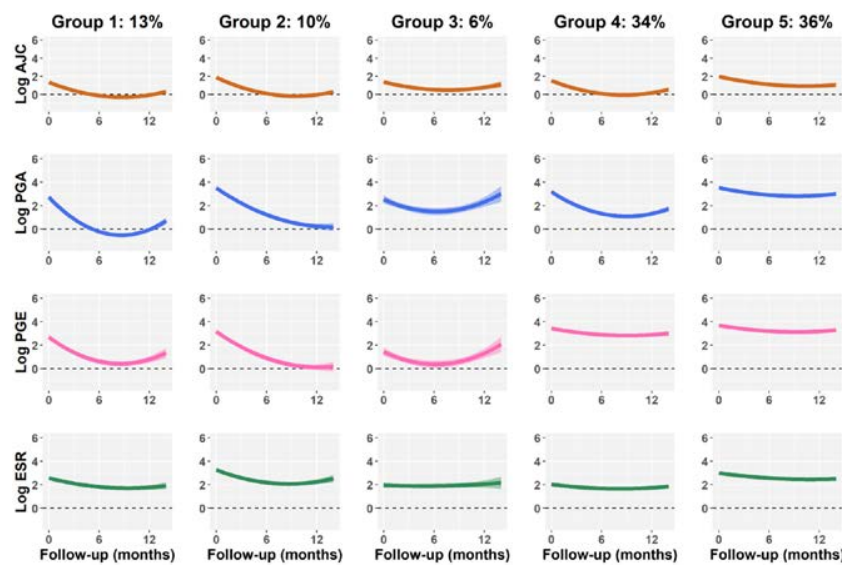

Figure 1. Clusters identified following ETN initiation in children and young people recruited to the UK BSPAR-ETN, BCRD, CAPS and CHARMS studies. 